\title{
Cerebral Perfusion Associates with Short-term Clinical Outcomes of Cerebral Venous Thrombosis
}

\author{
Yawei Wu${ }^{1}$, Jing Ye ${ }^{1 *}$, Hongmei Li ${ }^{1}$, Weiqiang Dou ${ }^{2}$, Lulu Liu ${ }^{1}$, Junhui Cai ${ }^{1}$, Hu Yuan ${ }^{1}$ and Xihai Zhao ${ }^{3}$ \\ ${ }^{1}$ Department of Radiology, Clinical Medical College of Yangzhou University, China \\ ${ }^{2}$ GE Healthcare, MR Research China, China
}

${ }^{3}$ Department of Biomedical Engineering, School of Medicine, Tsinghua University, China

*Corresponding author: Jing Ye, Department of Radiology, Clinical Medical College of Yangzhou University, Northern Jiangsu People's Hospital, Yangzhou, 225001, China.

Received Date: September 22, 2020

Published Date: October 15, 2020

\begin{abstract}
Background: To investigate the predictive value of Arterial Spin Labeling (ASL) perfusion imaging for short-term clinical outcomes of cerebral venous thrombosis (CVT) patients.
\end{abstract}

Materials and methods: Patients diagnosed with CVT by contrast-enhanced magnetic resonance venography (CE-MRV) were recruited and underwent ASL imaging before anticoagulation treatment. The cerebral blood flow (CBF) values in parenchymal abnormalities and the contralateral mirror areas were measured and the ratio of $\mathrm{CBF}(\mathrm{rCBF})$ between these two areas was calculated. All patients were followed-up within 3 weeks after treatment and the adverse clinical outcome was recorded. The association between rCBF and adverse clinical outcome was analyzed using logistic regression. The receiver-operating-characteristic (ROC) analysis was utilized to determine the area-under-the-curve (AUC) of rCBF in discriminating adverse clinical outcome.

Results: Twenty-three patients (mean age 42.09 \pm 14.71 years; 14 females) were enrolled, of which 7 (30.43\%) developed adverse clinical outcomes within 3 weeks. Patients with adverse clinical outcome showed significantly lower rCBF than those without $(0.54 \pm 0.17$ vs. $0.83 \pm 0.18$, $\mathrm{P}=0.002$ ). The $\mathrm{rCBF}$ was significantly associated with adverse clinical outcome before (odds ration [OR] $=0.207,95 \%$ confidence interval [CI] 0.049 $0.879, \mathrm{P}=0.033$ ) and after ( $\mathrm{OR}=0.167,95 \% \mathrm{CI} 0.030-0.914, \mathrm{P}=0.039$ ) adjusting for age and gender. ROC curve analysis indicated that patients with $\mathrm{rCBF}<65.2 \%$ were more likely to have adverse clinical outcome (AUC=0.893, sensitivity $87.5 \%$, specificity $85.7 \%$ ).

Conclusion: The cerebral perfusion determined by ASL in CVT patients is associated with short-term adverse clinical outcome, suggesting that baseline cerebral perfusion might be an effective predictor for prognosis in CVT patients.

Keywords: Cerebral venous thrombosis; Cerebral perfusion; Arterial spin labeling; Adverse outcome; Prognosis

\section{Introduction}

Cerebral venous thrombosis (CVT) is a relatively rare cerebrovascular disease which is accounting for $0.5-1 \%$ of all strokes and most commonly seen in young women $[1,2]$. After early anticoagulation treatment with heparin, parenchymal damage can be largely recovered in most of CVT patients. However, serious or life-threating consequence still happens in some CVT patients $[3,4]$. As such, it is important to identify the CVT patients who are at high risk of developing adverse clinical outcome. Few studies reported the potential risk factors for adverse clinical outcome of CVT. An animal experiment conducted by Nakase et al showed that regional cerebral blood flow (CBF) and oxygen saturation could predict 
clinical outcome of CVT [5]. It is well demonstrated that the extent of parenchymal damage can be evaluated by CBF measurement [6]. Gupta et al utilized Computed tomography perfusion (CTP) to measure the cerebral perfusion for $20 \mathrm{CVT}$ patients and found that the relative $\mathrm{CBF}$ ( $\mathrm{rCBF}$ ) of the parenchymal abnormalities to contralateral regions in the contralateral hemisphere was significantly associated with adverse clinical outcome [7]. However, most of previous studies utilized CTP with administration of iodine-based contrast agent which is not applicable for patients with allergy to iodine or renal dysfunction. Arterial spin labeling (ASL) magnetic resonance perfusion imaging technique has become an alternative approach for assessing cerebral perfusion in clinical settings due to its advantages of no radiation and contrast agent free but similar performance with CTP [8]. Most recently, Furuya et al successfully evaluated the therapeutic course of 2 CVT patients by serial ASL [9].

Although routine MRI combined with magnetic resonance venography (MRV) has been considered as the gold standard for the diagnosis of CVT [10], the thrombosis in cortical veins and bridging veins could not be clearly displayed. In CVT patients, thrombosis can involve cortical and bridging veins and subsequently induce parenchymal damage and worsen the clinical condition due to the reduced perfusion, such as venous infarction and hemorrhage transformation $[5,11,12]$. The alternation of perfusion changes in CVT patients may reflect both venous congestion and the reduction of cerebral blood flow. Previous studies on CVT patients which involves cerebral perfusion information all showed perfusion abnormalities of parenchymal lesions [7,9,13,14]. Therefore, perfusion imaging may be helpful for the diagnosis and localization of CVT. The purpose of this study was to investigate the characteristics of cerebral perfusion by ASL and their predictive value for short-term clinical outcome in CVT patients.

\section{Materials and Methods}

\section{Study population}

Patients who were diagnosed with CVT by contrast-enhanced magnetic resonance venography (CE-MRV) were enrolled in this study. Routine brain MR imaging and ASL imaging for brain were performed for each subject after recruitment before treatment. The exclusion criteria are: 1) history of cerebral venous thrombosis in the past 6 months; 2) cerebral neoplasm; 3) conditions which can alter the perfusion parameters, such as intracranial stenosis and arteriovenous malformations. This study was approved by local institutional review board and written informed consent was obtained from each patient.

\section{MR Imaging protocols}

All MRI examinations were conducted on 3T MRI scanner (GE Medical Systems, Discovery 750 and 750W, Milwaukee, USA) with an 8-channel receiving head coil. All patients were well trained before MR examinations to keep their heads still during the scan. CE-MRV was performed using GRE sequence, parameters as fol- lows: repeat time (TR)/echo time (TE) 3.5/1.4 ms, field of view (FOV) $240 \times 240 \mathrm{~mm}^{2}$, flip angle $20^{\circ}$, slice thickness $2 \mathrm{~mm}$. Routine brain imaging protocol was acquired with the following sequences: T1-weighted (T1W) (TR/TE 2534/19 ms, FOV $240 \times 240$ $\mathrm{mm}^{2}$, slice thickness $5 \mathrm{~mm}$, and number of slices 22); T2-weighted (T2W) (TR/TE 5325/101 ms, FOV $240 \times 240 \mathrm{~mm}^{2}$, slice thickness $5 \mathrm{~mm}$, and number of slices 22); and Diffusion weighted imaging (DWI) (TR/TE $4488 / 79.5 \mathrm{~ms}$, FOV $240 \times 240 \mathrm{~mm}^{2}$, slice thickness $5 \mathrm{~mm}$, and number of slices 22). Perfusion-weighted images were obtained by acquiring 3D pCASL sequence. In addition to the background suppression pulses within the slab, inferior saturation pulses were applied to suppress the inflowing arterial blood spins. The imaging parameters for 3D pCASL were as follows: 3D spiral fast spin echo (FSE) sequence, TR $4844 \mathrm{~ms}$, TE $10.5 \mathrm{~ms}$, FOV $240 \times 240$ $\mathrm{mm}^{2}$, slice thickness $4 \mathrm{~mm}$, slice gap $0 \mathrm{~mm}$, number of slices 36,512 sampling points on eight spiral arms, spatial resolution $3.64 \mathrm{~mm}$, bandwidth $62.5 \mathrm{kHz}$, NEX 2, post labeling delay (PLD) $2025 \mathrm{~ms}$, and labeling duration $2000 \mathrm{~ms}$. No motion correction and slice timing correction were performed.

\section{Clinical follow-up}

The follow-up MRI was conducted for brain within 3 weeks after anticoagulation treatment. The routine brain imaging protocol was performed for the follow-up examination. The adverse clinical outcome in this study was recorded which is defined as: 1) enlargement of parenchymal lesions; or 2) cerebral hemorrhagic transformation.

\section{MR image analysis}

The ASL perfusion images were transferred to Advantage Workstation 4.6 (GE medical systems) with dedicated software of 3D-ASL with single compartment model assumed. Subsequent quantifications were performed according to the following equation:

where $\mathrm{T}_{1 \mathrm{~b}}$ was defined as for the $\mathrm{T} 1 \mathrm{of}$ the blood $(1600 \mathrm{~ms}), \mathrm{T}_{1 \mathrm{~g}}$ as the T1 of the grey matter (1200 ms), $\alpha$ as the labeling efficiency $(0.8), \lambda$ as the cortex-blood partition coefficient ( 0.9$), t_{\text {sat }}$ as the time of saturation performed before imaging $(2000 \mathrm{~ms}), \tau$ as the labeling duration (2025 ms), and PLD as the postlabeling delay time [15]. ${ }^{15}$ In addition, proton density weighted images, denoted as PD here, were directly acquired during ASL image acquisition. A moderate image smoothing was applied by using $3 \times 3$ convolutional kernel.

Cerebral blood flow (CBF) was measured by 2 experienced radiologists ( $>3$ years' experience in neuroimaging). The regions of interest (ROIs) were placed at the areas of parenchymal abnormalities characterized by hyperintense on T2W and DWI images and the contralateral regions (normal reference) in contralateral hemisphere. The CBF of ROIs was measured for 2 times by one radiologist. The mean values of $\mathrm{CBF}$ and the ratio of $\mathrm{CBF}(\mathrm{rCBF}=\mathrm{CBF}$ of parenchymal abnormalities / CBF of contralateral regions) between the measurements of 2 times were calculated for statistical analysis. 


\section{Reproducibility of ASL measurements}

All patients were selected for testing the inter-observer and intra-observer reproducibility in measuring CBF. Two radiologists independently measured the $\mathrm{CBF}$ values of the whole ROIs of parenchymal abnormalities and contralateral normal hemisphere on ASL images for all patients. One radiologist performed a repeat measurement of the CBF values of the whole ROIs in above cerebral regions on ASL images with a time interval of 3 months to minimize the memory bias.

\section{Statistical analysis}

All statistical analyses were performed by SPSS (V.19.0; SPSS Inc, Chicago, Illinois, USA). Kolmogorov-Smirnov test was applied to estimate the normality of all the measurements. The continuous variables were presented as mean \pm standard deviation (SD) and the categorical variables were presented as frequency. The absolute $\mathrm{CBF}$ values of grey matter, white matter, and whole ROIs were compared between parenchymal abnormalities and the contralateral regions with paired $t$ test. Baseline clinical characteristics, cerebral absolute CBF values and $\mathrm{rCBF}$ were compared between patients with and without adverse clinical outcome using independent $t$ test, Mann-Whitney U test, or Chi-square when appropriate. Logistic regression was used to calculate the odds ratio (OR) and 95\% confidence interval (CI) of $\mathrm{rCBF}$ in predicting adverse clinical outcome before and after adjusting for confounding factors. Grubbs' outlier test was performed before logistic regression. The receiver-operating-characteristic analysis was utilized to determine the area-under-the-curve (AUC) of rCBF in discriminating adverse clinical outcome. The intraclass correlation coefficient (ICC) and corresponding $95 \% \mathrm{CI}$ of $\mathrm{rCBF}$ were calculated to determine the inter-observer and intra-observer agreement. A P value $<0.05$ was considered as statistically significant.

\section{Results}

In total, 23 patients (mean age $42.09 \pm 14.71$ years; 14 females) with CVT were included in this study. Of the 23 patients, 17 (73.91\%) presented with headache and $1(4.35 \%)$ was postpartum at baseline. The potential causes of CVT of this study population included oral contraceptives for a long time $(n=6)$, infections $(n=5)$, puerperium $(n=1)$, nephrotic syndrome $(n=1)$, and sicca syndrome $(\mathrm{n}=1)$. The potential cause for the rest 9 patients is unknown. During 3 weeks' follow-up, 7 (30.43\%) patients developed adverse clinical outcomes, including $4(17.39 \%)$ patients with enlargement of parenchymal lesions and $3(13.04 \%)$ patients with both hemorrhagic transformation and enlargement of parenchymal lesions.

\section{Imaging characteristics on MRV and ASL images}

On MRV images, CVT can be found in 15 (65.22\%), 17 (73.91\%), $5(21.74 \%)$, and $3(13.04 \%)$ patients in sagittal superior sinus, transverse and sigmoid sinus, straight sinus, and cortical veins, respectively. Of the 23 patients, 9 (39.13\%), 12 (52.17\%) and 2 $(8.70 \%)$ were found to have CVT in 1, 2, and $>2$ regions, respectively. In this study population, all ASL perfusion abnormalities were localized adjacent to the occluded sinus. Of all 23 patients, 20 $(86.96 \%)$ showed hypoperfusion and $3(13.04 \%)$ showed hyperperfusion in parenchymal abnormalities compared to contralateral regions. Paired $t$ test showed that the absolute CBF of the parenchymal abnormalities was significantly lower than that of contralateral regions $(33.98 \pm 13.52 \mathrm{~mL} / 100 \mathrm{~g} / \mathrm{min}$ vs. $46.69 \pm 14.10 \mathrm{~mL} / 100 \mathrm{~g} /$ $\min , \mathrm{P}=0.003)$. There were no significant differences in the absolute CBF values of parenchymal abnormalities (37.21 \pm 20.19 $\mathrm{mL} / 100 \mathrm{~g} / \mathrm{min}$ vs. $26.79 \pm 6.15 \mathrm{~mL} / 100 \mathrm{~g} / \mathrm{min}, \mathrm{P}=0.200$ ) between patients with good and adverse clinical outcome. Patients with adverse clinical outcome showed significantly lower $\mathrm{rCBF}(0.54 \pm 0.17$ vs. $0.83 \pm 0.18, \mathrm{P}=0.002$ ) compared to those with good clinical outcome (Figure 1\&2).

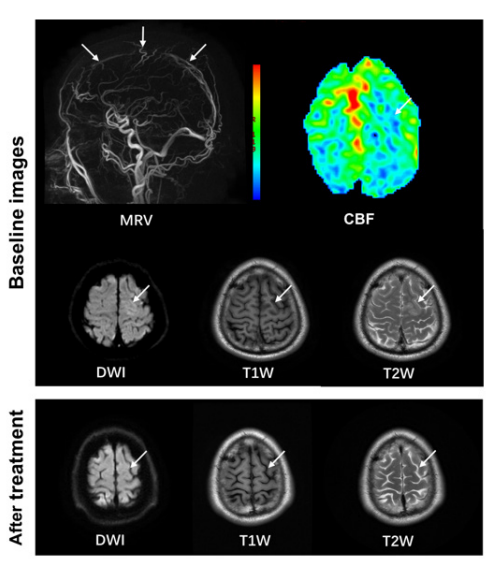

Figure 1: A 43y male patient with CVT who had good clinical outcome. CE-MRV showed multiple stenosis in the sagittal sinus due to thrombosis (arrows). Slight hyperintense can be found on DWI and T2W images (arrows). The rCBF at baseline was 0.743 and the follow-up images after 15-day treatment showed that the parenchymal abnormalities were reversed (arrows). Figure 1: A 43y male patient with CVT who had good clinical outcome. CE-MRV showed multiple stenosis in the sagittal sinus due to thrombosis (arrows). Slight hyperintense can be found on DWI and T2W images (arrows). The rCBF at baseline was 0.743 and the follow-up images after 15-day treatment showed that the parenchymal abnormalities were reversed (arrows). 

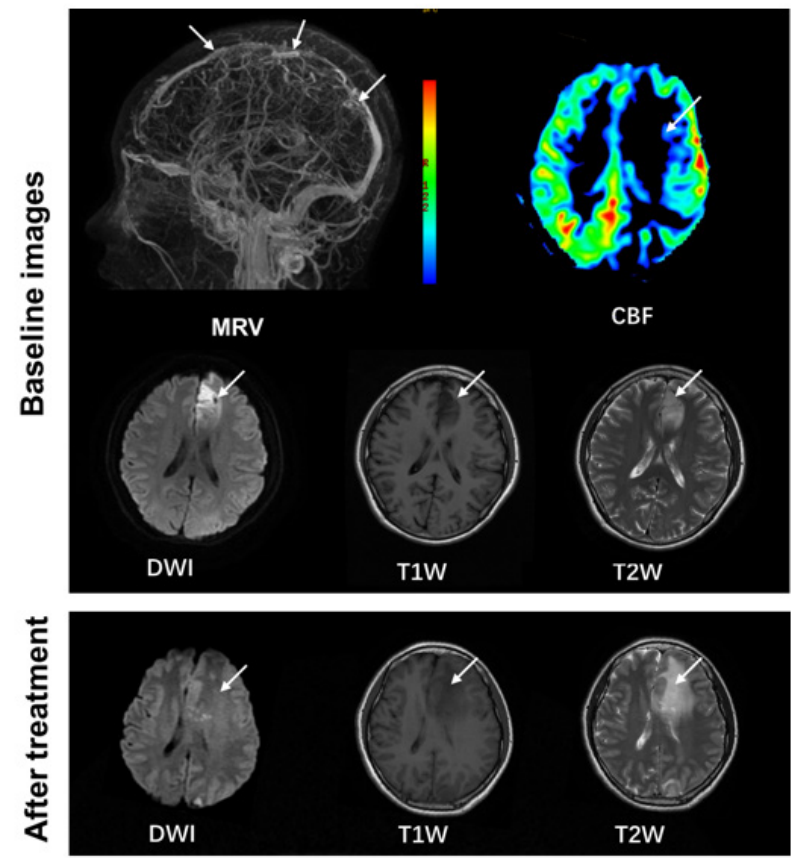

Figure 2: A 45y female patient with CVT who had adverse clinical outcome. CE-MRV showed multiple stenosis in the sagittal sinus due to thrombosis (arrows). Hyperintense can be found on DWI and T2W images (arrows). The rCBF at baseline was 0.316 and the follow-up images after 5-day treatment showed significant enlargement of the parenchymal abnormalities compared with baseline (arrows).

\section{Association between rCBF and adverse clinical outcome}

No outlier was found by Grubbs' outlier test. After that, logistic regression analysis demonstrated that $\mathrm{rCBF}$ of the whole ROIs was significantly associated with adverse clinical outcome before (OR = $0.207,95 \%$ CI $0.049-0.879, \mathrm{P}=0.033$ ) and after (OR $=0.167,95 \%$
CI $0.030-0.914, \mathrm{P}=0.039$ ) adjusting for age and gender with increment of $10 \%$ of rCBF. ROC curve analysis indicated that patients with $\mathrm{rCBF}<65.2 \%$ (optimal cut-off value) were more likely to have adverse clinical outcome (AUC $=0.893$, sensitivity $87.5 \%$, specificity $85.7 \%$, Figure 3).

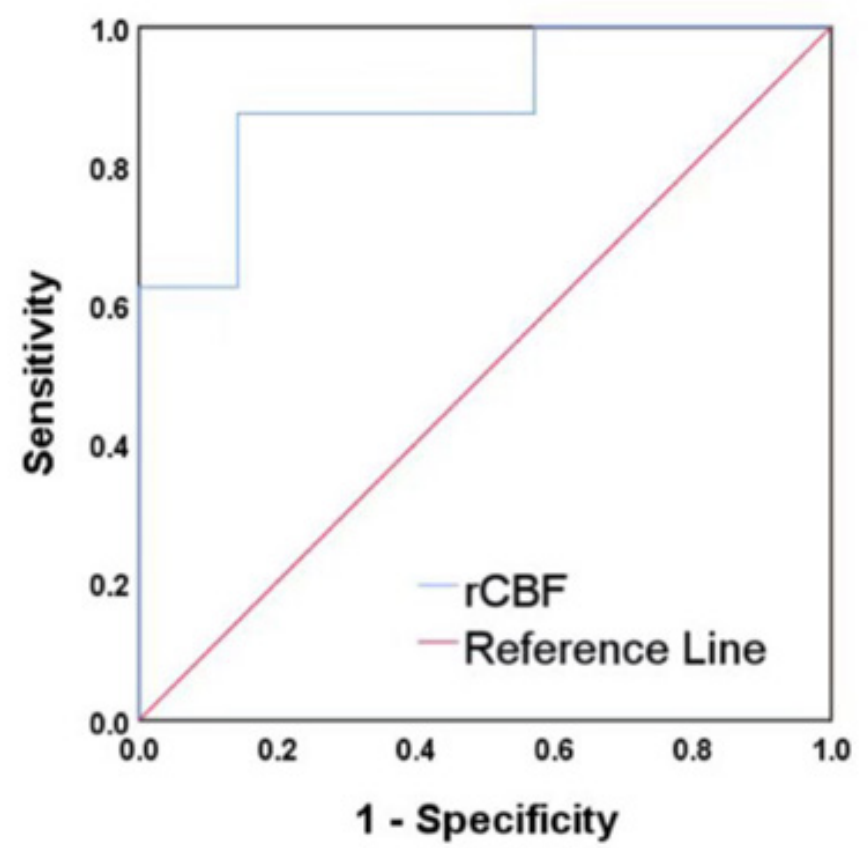

Figure 3: ROC-curve of the rCBF measured by ASL predicting adverse clinical outcome. 


\section{Reproducibility of ASL measurements}

Excellent intra-observer (ICC, 0.964; 95\%CI, 0.917-0.985) and inter-observer (ICC, 0.936; 95\%CI, 0.857-0.972) agreements were found in measuring rCBF using ASL.

\section{Discussion}

This study investigated the characteristics of cerebral perfusion in cerebral parenchymal abnormalities of CVT patients before anticoagulation treatment using ASL and their predictive value for short-term adverse clinical outcome. We found that patients with adverse clinical outcome had significantly lower rCBF compared with those without. Logistic regression analysis revealed that $\mathrm{rCBF}$ was independently associated with adverse clinical outcome. Our findings suggested that the perfusion determined by ASL before anticoagulation treatment might be an effective predictor for shortterm adverse clinical outcome in patients with CVT. In this study, most of the CVT patients $(20 / 23)$ had lower perfusion in parenchymal abnormalities compared to contralateral regions. Similar results were reported in a study by Kang et al (10/13) [13]. Even though the cerebral collateral pathway is sufficient, cerebral venous thrombosis will lead to intracerebral venous congestion and the increase of venous pressure, which may also lower the perfusion pressure $[16,17]$. In our study population, 3 patients showed relative hyperperfusion in the parenchymal lesions which may be due to the accumulation of acidic metabolites that result in vasodilation in the abnormal area or the physiological compensation of reserved capillaries in the early period of CVT.

In this study, we found that the baseline rCBF in patients with adverse clinical outcome was significantly lower than that in those patients without and lower rCBF could effectively predict adverse clinical outcome in CVT patients. Nakase et al reported that the extent of CBF reduction reflected the subsequent parenchymal damage in CVT patients [6]. The causes of arterial stroke and cerebral venous infarction are different. In arterial stroke, the occlusion of feeding artery directly leads to the reduction of cerebral perfusion. In contrast, cerebral venous infarction was commonly caused by cerebral venous thrombosis [18]. The reduced CBF may lead to functional disturbance of brain tissue in both cerebral events [19]. Pathophysiologically, in arterial stroke, decrease in CBF can induce the lack of glucose and oxygen, in turn, the energy failure leads to a cascade of the depletion of adenosine triphosphate (ATP) and a decrease in sodium-potassium adenosine triphosphatase $\left(\mathrm{Na}^{+}-\mathrm{K}^{+} \mathrm{AT}\right.$ Pase) activity [20]. In contrast, the fact of pathophysiology of CVT is a multistep evolution and the time course of parenchymal damage is prolonged, which can persist for a long time but still may be reversible $[12,19]$. Whether the damage of cerebral parenchymal is reversible depends on the extent of reduction in cerebral perfusion. It might be plausible that the anticoagulation drug does not get to be delivered to the thrombus effectively when perfusion is lowered to a certain degree. A previous study on arterial stroke conducted by Shimosegawa et al showed that completed infarction induced by $40 \%$ reduction of $\mathrm{CBF}$, but no such threshold has been reported in CVT patients [21]. Therefore, we assumed that this threshold may be also suitable for patients with CVT. Gupta et al determined the pre-treatment measurements in the lesions and contralateral regions by CTP in CVT patients and found that rCBF was the best parameter to evaluate the clinical outcome with the optimal $\mathrm{rCBF}$ cut-off value of $60.5 \%$ (AUC $=0.755$, sensitivity $68.2 \%$, specificity $66.7 \%$ ) [7]. This optimal cut-off value is consistent with the results in arterial stroke to some extent. The optimal cut-off value of $\mathrm{rCBF}$ in the present study is slightly higher than the literature report which might be due to the limited sample size.

A single PLD of $2000 \mathrm{~ms}$ was recommended as a standard clinical protocol for adult population, which is a robust and straightford means of obtaining reliable CBF images [22]. However, the difference in individual PLD and inappropriate PLD will attenuate the accuracy of CBF measurement. Both the arterial transit time (ATT) and CBF can be accurately estimated when serial ASL images were acquired with multiple PLDs $[22,23]$. Few previous studies utilized ASL to assess cerebral perfusion in CVT patients. There might be different in the mechanism of cerebral ischemia between arterial occlusion and venous congestion. Furuya et al conducted ASL imaging with both PLDs of $1000 \mathrm{~ms}$ and $2200 \mathrm{~ms}$, despite the scan parameters were different from the present study, they found that shorter PLD of 1000 ms couldn't reflect the different perfusion between parenchymal abnormalities and contralateral regions in CVT patients [9]. Accordingly, a moderate PLD of 2025 ms was used in the present study. This may be a limitation of this study. It might be ideal to use multiple PLD times in ASL imaging for accurate quantification of CBF and transit time in future studies. Beyond single PLD, other limitations should be noted in this study. First, we measured $\mathrm{rCBF}$ by drawing the ROIs according to the hyperintense on DWI which may represent the core area of infarction. Although a study by Gupta et al demonstrated that there was no significant difference in cerebral perfusion between core and periphery regions [7] which may represent penumbra in parenchymal abnormalities, it might be interesting to evaluate the characteristics of CBF in periphery regions of parenchymal abnormalities. Second, in the present study, only the most severe slice with maximum area of ischemia was selected for measuring rCBF in each patient. The selected slice may not represent the overall lesion. Finally, the sample size of the present study is limited. Larger patient population is suggested to be included in future studies.

\section{Conclusion}

In conclusion, the cerebral perfusion determined by ASL in CVT patients is independently associated with short-term adverse clinical outcome, suggesting that baseline cerebral perfusion might be an effective predictor for prognosis in CVT patients.

\section{Acknowledgments}

None. 


\section{Disclosure of Interest}

The authors declare that they have no competing interest.

\section{References}

1. Zuurbier SM, Middeldorp S, Stam J, Coutinho JM (2016) Sex differences in cerebral venous thrombosis: A systematic analysis of a shift over time. Int J Stroke 11(2): 164-170.

2. Stam J (2005) Thrombosis of the Cerebral Veins and Sinuses. N Engl J Med 352(17): 1791-1798.

3. Zhou LX, Yao M, Cui LY, Li ML, Zhu YC, et al. (2017) The Structural Imaging Characteristics and Its Clinical Relevance in Patients with Cerebral Venous Thrombosis-A Retrospective Analysis from One Single Center in China. Front Neurol 8: 648.

4. Gulati D, Strbian D, Sundararajan S (2014) Cerebral venous thrombosis diagnosis and management (Article). Stroke 45: e16-e18.

5. Nakase H, Heimann A, Kempski O (1996) Alterations of regional cerebral blood flow and oxygen saturation in a rat sinus-vein thrombosis model. Stroke 27(4): 720-727.

6. Nakase H, Kempski OS, Heimann A, Takeshima T, Tintera J (1997) Microcirculation after cerebral venous occlusions as assessed by laser Doppler scanning. J Neurosurg 87(2): 307-314.

7. Gupta RK, Bapuraj JR, Khandelwal N, Khurana D (2014) Prognostic indices for cerebral venous thrombosis on CT perfusion: a prospective study. Eur J Radiol 83(1): 185-190.

8. Bivard A, Krishnamurthy V, Stanwell P, Levi C, Spratt NJ, et al. (2014) Arterial spin labeling versus bolus-tracking perfusion in hyperacute stroke. Stroke 45(1): 127-133.

9. Furuya S, Kawabori M, Fujima N, Tokairin K, Goto S, et al. (2017) Serial Arterial Spin Labeling May Be Useful in Assessing the Therapeutic Course of Cerebral Venous Thrombosis: Case Reports. Neurol Med Chir (Tokyo) 57(10): 557-561.

10. Kawai N, Shindou A, Masada T, Tamiya T, Nagao S (2005) Hemodynamic and metabolic changes in a patient with cerebral venous sinus thrombosis: evaluation using 0-15 positron emission tomography. Clin Nucl Med 30(6): 391-394.

11. Bergui M, Bradac GB, Daniele D (1999) Brain lesions due to cerebral venous thrombosis do not correlate with sinus involvement Neuroradiology 41(6): 419-424.

12. Fries G, Wallenfang T, Hennen J Velthaus M, Heimann A, Schild H et al. (1992) Occlusion of the pig superior sagittal sinus, bridging and cortical veins: multistep evolution of sinus-vein thrombosis. J Neurosurg 77(1) 127-133.
13. Kang JH, Yun TJ, Yoo RE, Yoon BW, Lee AL, et al. (2017) Bright sinus appearance on arterial spin labeling MR imaging aids to identify cerebral venous thrombosis. Medicine 96(41): e8244.

14. Mokin M, Ciambella CC, Masud MW, Levy EI, Snyder KV, et al. (2016) Whole-Brain Computed Tomographic Perfusion Imaging in Acute Cerebral Venous Sinus Thrombosis. Interv Neurol 4(3-4): 104-112.

15. Wang T, Li Y, Guo X, Huang D, Ma L, et al. (2016) Reduced perfusion in normal-appearing white matter in mild to moderate hypertension as revealed by 3D pseudocontinuous arterial spin labeling. J Magn Reson Imaging 43(3): 635-643.

16. Schaller B, Graf R, Sanada Y, Tolnay M, Rosner G, et al. (2003) Hemodynamic changes after occlusion of the posterior superior sagittal sinus: an experimental PET study in cats. AJNR Am J Neuroradiol 24(9): 1876-1880.

17. Leach JL, Fortuna RB, Jones BV, Gaskill-Shipley MF (2006) Imaging of cerebral venous thrombosis: current techniques, spectrum of findings, and diagnostic pitfalls. Radiographics 26: S19-S43.

18. Wang W, Lin C, Hong J, Wang S, Gu J (2018) Effects of Increased Intracranial Pressure Gradient on Cerebral Venous Infarction in Rabbits. World Neurosurg 120: e161-e168.

19. Doege CA, Tavakolian R, Kerskens CM, Romero BI, Lehmann R, Einhäupl KM, Villringer A (2001) Perfusion and diffusion magnetic resonance imaging in human cerebral venous thrombosis. J Neurol 248(7): 564571.

20. Kulik T, Kusano Y, Aronhime S, Sandler AL, Winn HR (2008) Regulation of cerebral vasculature in normal and ischemic brain. Neuropharmacology 55(3): 281-288.

21. Shimosegawa E, Hatazawa J, Inugami A, Fujita H, Ogawa T, Aizawa Y et al. (1994) Cerebral infarction within six hours of onset: prediction of completed infarction with technetium-99m-HMPAO SPECT. J Nucl Med 35(7): 1097-1103.

22. Alsop DC, Detre JA, Golay X, Günther M, Hendrikse J, HernandezGarcia L et al. (2015) Recommended implementation of arterial spinlabeled perfusion MRI for clinical applications: A consensus of the ISMRM perfusion study group and the European consortium for ASL in dementia. Magn Reson Med 73(1): 102-116.

23. Cohen AD, Agarwal M, Jagra AS, Nencka AS, Meier TB, Lebel RM et al. (2019) Longitudinal Reproducibility of MR Perfusion Using 3D Pseudocontinuous Arterial Spin Labeling with Hadamard-Encoded Multiple Postlabeling Delays. J Magn Reson Imaging: 30. 